\title{
Novel gene $h$ Biot 2 is an independent prognostic factor in colorectal cancer patients
}

\author{
YANG-MEI SHEN $^{1,3}$, GUNNAR ARBMAN $^{4}$, PER SANDSTRÖM $^{2}$, PER GULLSTRAND ${ }^{2}$, YU-QUAN WEI $^{5}$, \\ HONG ZHANG $^{6}$, JOHAN ROSELL ${ }^{1}$, BIRGIT OLSSON ${ }^{1}$, FENG PENG $^{5}$, HAN-SHUO YANG $^{5}$, \\ CHUN-TING WANG ${ }^{5}$ and XIAO-FENG SUN ${ }^{1,7}$
}

\begin{abstract}
Departments of ${ }^{1}$ Oncology and ${ }^{2}$ Surgery, Institute of Biomedicine and Surgery, University of Linköping, Linköping, Sweden; ${ }^{3}$ Department of Pathology, West China Second University Hospital, Sichuan University, Chengdu, P.R. China; ${ }^{4}$ Department of Surgery, Vrinnevi Hospital, Norrköping, Sweden; ${ }^{5}$ State Key Laboratory of Biotherapy of Human Diseases, West China University Hospital, Sichuan University, Chengdu, P.R. China; ${ }^{6}$ Division of Tumor Biology, Systems Biology Research Centre, University of Skövde, Sweden; ${ }^{7}$ Department of Gastrointestinal Surgery, West China Hospital, Sichuan University, Chengdu, P.R. China
\end{abstract}

Received February 22, 2011; Accepted April 14, 2011

DOI: $10.3892 /$ or.2011.1521

\begin{abstract}
The present study investigated the expression of the novel gene $h$ Biot 2 in colorectal cancer (CRC) and its relationships with clinicopathological variables in CRC patients. The expression of $h$ Biot 2 in 163 primary CRCs together with the corresponding normal mucosa, 36 liver metastases and 5 colon cancer cell lines was examined using real-time PCR. In situ hybridization (ISH) was performed to evaluate the localization of $h \mathrm{Biot} 2$ expression in CRC and normal mucosa. $h$ Biot 2 expression at the RNA level was localized in the nucleus of tumor cells and normal epithelial cells. The mean expression of $h$ Biot 2 in the CRCs $(243.571 \pm 564.569)$ was higher compared to the normal mucosa $(107.252 \pm 413.635$, $\mathrm{P}<0.0001)$ and liver metastasis samples $(42.002 \pm 40.809$, $\mathrm{P}=0.0002)$. $h$ Biot 2 expression was increased from stages $\mathrm{I}+$ II to III $(\mathrm{P}=0.047)$, and no difference in the expression was found in stages III and IV $(\mathrm{P}=0.452)$. A high value of $h$ Biot 2 was associated with a poorer prognosis compared with a low value independently of gender, age, tumor site, stage and differentiation ( $\mathrm{P}=0.007, \mathrm{RR} 7.519,95 \%$ CI 1.729-32.704). Liver metastasis, smaller tumors, non-local recurrence and primary liver surgery alone were associated with a higher value of $h$ Biot 2 compared to larger tumors, local recurrence and repeated liver surgery $(\mathrm{P}=0.003,0.044$ and 0.026 , respectively). An inverse relationship was found between $h$ Biot2 expression and the metastatic potential of the colon cancer
\end{abstract}

Correspondence to: Professor Xiao-Feng Sun, Department of Oncology, Institute of Biomedicine and Surgery, University of Linköping, S-581 85 Linköping, Sweden

E-mail: xiao-feng.sun@liu.se

Key words: human Biot2, colorectal cancer, prognosis, real-time $\mathrm{PCR}$, in situ hybridization cell lines. Thus, increased expression of $h$ Biot 2 may be an early and interim event in the development of CRC. A higher expression of $h$ Biot 2 in primary CRC patients independently indicates a poorer prognosis.

\section{Introduction}

Colorectal cancer (CRC) is the third most common cancer in the world and the second most frequent cause of cancerrelated deaths (1). In 2007, approximately 1.2 million people were diagnosed around the world. In Sweden, CRC is the third most common form of cancer among men and women with almost 6,000 new cases every year (2). CRC is characterized by an unknown genetic heterogeneity with new genes and pathways involved in tumor genesis continually emerging.

Molecular events resulting in progression of CRC are complex and poorly understood. Furthermore, discovery of novel molecular markers has facilitated understanding of the molecular and cellular mechanisms underlying the development and progression of CRC (3-5). It would be beneficial to identify CRC risk factors and novel molecular markers that could help both in early diagnosis and treatment of the disease.

The novel gene $h$ Biot2 (human Biot2) is a homologous gene of $r$ Biot2 (rat Biot2) that was identified using rabbit serum immunized with human mammary cancer and ovarian cancer cells to screen the rat testis cDNA expression library using the serological analysis of the recombinant cDNA expression library (SEREX) approach (6). $h$ Biot 2 is located in chromosome 10 (10p11.22) with $1819 \mathrm{bp}$. Bioinformatic analysis and previous studies suggest that $r \operatorname{Biot} 2$ might be involved in sperm development in the rat $(7,8)$, and $h \operatorname{Biot} 2$ was found to have the potential to stimulate cell proliferation in human endometrial cancer (9). Therefore, it is presumed that $h$ Biot2 plays an important role in carcinogenesis.

The present study is the first to investigate $h \operatorname{Biot} 2$ expression in primary CRC along with the corresponding normal 
mucosa, liver metastases and colon cancer cell lines, as well as the relationships of the $h$ Biot 2 expression with clinicopathological variables in CRC patients. The results may help us to improve the understanding of the possible role of $h \mathrm{Biot} 2$ in the development and aggressiveness of CRC.

\section{Materials and methods}

Patients. The study included 163 primary CRCs, along with the corresponding normal mucosa, and 36 liver metastasis samples of CRC from Linköping University Hospital between 1990 and 2009. In each patient, the primary tumor and the corresponding normal colonic mucosa were collected for comparison. All specimens were flash-frozen in liquid nitrogen and then stored at $-80^{\circ} \mathrm{C}$. According to the histological diagnostic criteria of the World Health Organization, all samples were examined by pathologists at the Department of Pathology at Linköping University to confirm their histopathologic type, stage and metastasis. The liver metastasis specimens were collected with or without primary CRC surgery. The patients were grouped in the present study according to the mean size of the liver metastases $(\leq$ or $>42 \mathrm{~mm}$ ), local recurrence at a previously resected area in the liver (yes or no), distant metastasis rather than in the liver (yes or no), and liver surgery (primary liver surgery or repeated surgery).

Data regarding expression of p73 $(n=21)$ and meningioma-associated protein (MAC30) $(n=29)$ determined by immunohistochemistry, was obtained from previous studies performed at our laboratory on the same material as used in the present study $(10,11)$. The small numbers of cases were due to the matched cases available with the present study.

Colon cell lines. Three human colon cancer cell lines, KM12C, KM12SM, KM12L4a, kindly provided by Professor I.J. Fidler (M.D. Anderson Cancer Center, Houston, TX) were used in the present study. The KM12C cell line was originally derived from a patient with a TNM II colon cancer. KM12SM is a spontaneous liver metastasis which arose from injections of KM12C into the caecum of nude mice (12). KM12L4a is an experimental liver metastasis repeatedly injected into the spleen and retrieved from the liver of nude mice (13). Therefore, KM12L4a had the highest metastasis potential while KM12C had the lowest. All of the cells were cultured in Eagle's minimal essential medium (MEM) with Earle's salts, L-glutamine and non-essential amino acids (Sigma-Aldrich, Stockholm, Sweden), supplemented with $1.5 \% \mathrm{NaHCO}_{3}, 1 \mathrm{mM}$ Na-pyruvate (Invitrogen, Carlsbad, CA), 1X MEM vitamin solution (Invitrogen), 5\% penicillin-streptomycin (Invitrogen) and 10\% FBS (Gibco, Invitrogen, Carlsbad, CA).

Two colon cancer cell lines, HCT-116 with either wildtype p53 (HCT-116 ${ }^{\mathrm{p} 53+/+}$ ) or mutated p53 with a 40 -amino acid truncation (HCT-116 p53-/ $^{\text {) }}$ (14), were a kind gift from Dr B. Vogelstein (Johns Hopkins University, Baltimore, MD). The cells were cultured in McCoy's 5A (Sigma-Aldrich, St. Louis, MO) supplemented with 10\% FBS, 1X PEST (Gibco) and $1.5 \mathrm{mM}$ L-glutamine (Gibco).

RNA extraction and cDNA preparation. Total RNA was extracted from 163 primary tumors and the corresponding normal mucosa, 36 liver metastases and 5 colon cell lines using the TRIzol reagent (Sigma) and the RNase extraction kit (75144, Qiagen) according to the manufacturer's instructions (15). The concentration, purity and integrity of RNA were measured by Nano Drop and Agilent Bioanalyzer. Only RNA with high quantity and quality was used, and reverse-transcribed using the High Capacity cDNA reverse transcription kit (Applied Biosystems, Foster City, CA). Total RNA $(10 \mu \mathrm{l})$ was reverse transcribed using the MultiScribe ${ }^{\mathrm{TM}}$ reverse transcriptase according to the manufacturer's instructions as described (16), without an RNase inhibitor in a final volume of $20 \mu \mathrm{l}$. The program followed was: $25^{\circ} \mathrm{C}$ for $10 \mathrm{~min}$, $37^{\circ} \mathrm{C}$ for $120 \mathrm{~min}, 85^{\circ} \mathrm{C}$ for $5 \mathrm{~min}$, and $4^{\circ} \mathrm{C}$ for $120 \mathrm{~min}$.

Real-time PCR. In this study, a standard curve was constructed from a mRNA sample of known concentration and used for extrapolating absolute quantitative information for mRNA targets of unknown concentrations. In order to compare samples across runs, the housekeeping gene human GAPDH (glyceraldehyde-3 phosphate dehydrogenase) was run for each sample with a concurrent standard curve. The expression value of $h$ Biot 2 was determined by qRT-PCR with TaqMan ${ }^{\mathrm{TM}}$ gene expression fast master mix in Applied Biosystems 7900HT Fast real-time PCR system and normalized to GAPDH. Primers and probes were from TaqMan gene expression assays for $h$ Biot2 (Assay ID Hs00376654_m1, Applied Biosystems) and GAPDH (4352934E, Applied Biosystems). All of the samples were performed in triplicates. The PCR amplification program included denaturing at $95^{\circ} \mathrm{C}$ for $20 \mathrm{sec}$. The amplification and quantification program was repeated 40 times at $95^{\circ} \mathrm{C}$ for $1 \mathrm{sec}$ and at $60^{\circ} \mathrm{C}$ for $20 \mathrm{sec}$. In addition, $\mathrm{ddH}_{2} \mathrm{O}$ and a minus RT product as the negative control were analyzed for every plate.

In the present study, three methods were used to express the data. The first was the mean expression value, i.e. $h$ Biot2 mean value expressed in different tissues, such as normal mucosa. Since the patients exhibited individual differences, the $h$ Biot 2 basal expression was different among the patients. When we studied the relationships between the $h$ Biot2 expression value in each patient and the clinicopathological variables, the relative value was used, which was the $h$ Biot2 expression value in the primary tumor divided by the $h$ Biot 2 expression value in the corresponding normal mucosa. The third method used was to divide a low expression group and a high expression group by using the lowest $25 \%$ expression value as a cut-off point to analyze the impact of the $h$ Biot 2 relative expression value on prognosis.

In situ hybridization (ISH) with LNA probes. In order to determine the localization of $h \operatorname{Biot} 2$ expression in the tumor and normal mucosa, we examined RNA expression of $h$ Biot2 by using ISH. All probes used in ISH were the ones with a novel modified nucleotide termed LNA (locked nucleic acidbased). LNA is a bicyclic high-affinity nucleotide analog that significantly improves hybridization properties of DNA oligonucleotides (17-19). The LNA DIG-labelled probes were designed and synthesized by Exigon Company. The sequences were as follows: $h$ Biot 2 gene probe/5DigN/CATCCCCAACA GATAAAGATAATT/3Dig_N/; positive control probe/ 5DigN/CTCATTGTAGAAGGTGTGGTGCCA; negative control probe, GTGTAACACGTCTATACGCCCA. 

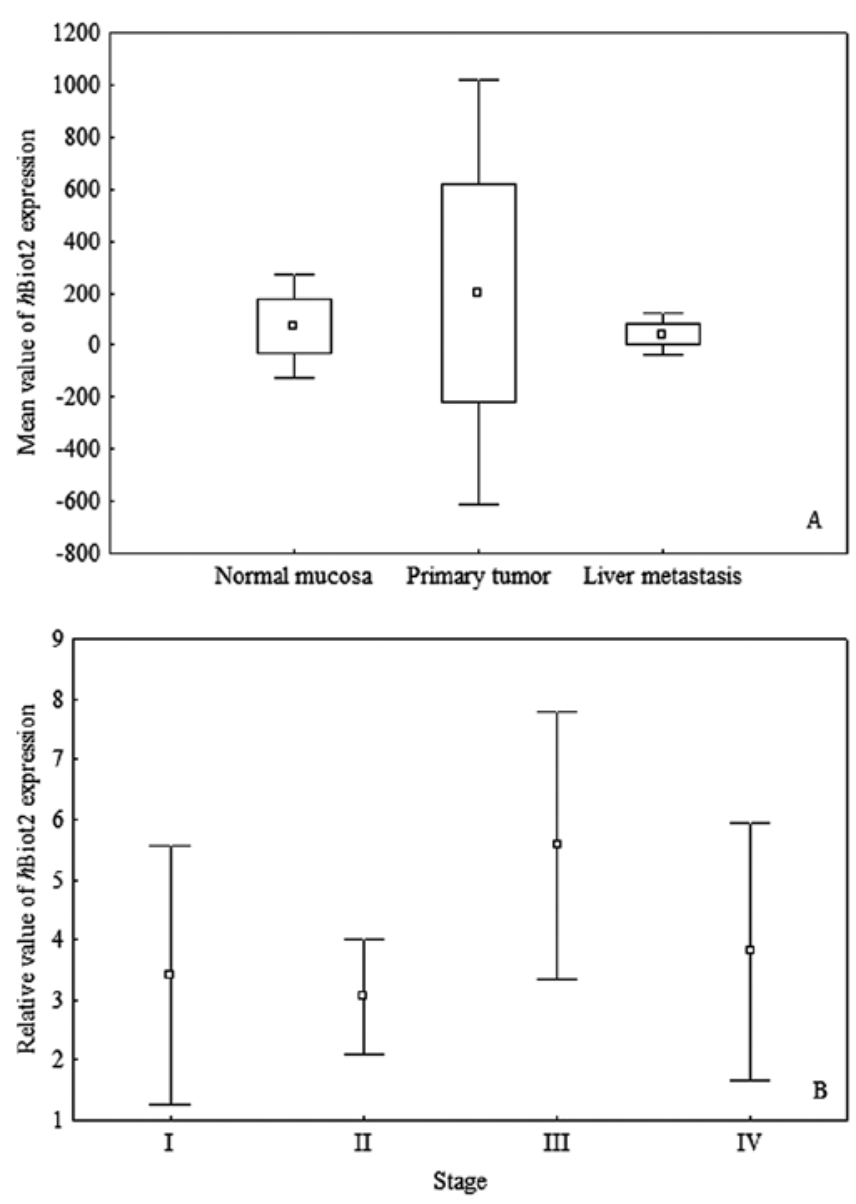

Figure 1 . The mean value of $h$ Biot 2 expression in the primary colorectal cancer samples $(243.571 \pm 564.569)$ was higher than that in the normal mucosa $(107.252 \pm 413.635, \mathrm{P}<0.0001)$ and liver metastasis $(42.002 \pm 40.809, \mathrm{P}=0.0002)$ (A). The relative value of $h$ Biot 2 expression ( $h$ Biot 2 expression value in the primary tumor divided by the $h$ Biot 2 expression value in the corresponding normal mucosa) in primary tumor patients was higher in stage III than in stage II (5.571 \pm 6.578 vs. $3.057 \pm 4.179, \mathrm{P}=0.018)$, while expression in stages I and II was very similar ( $3.413 \pm 4.580$ vs. $3.057 \pm 4.179, \mathrm{P}=0.368)$. Stage IV had lower expression than stage III but the difference was not significant (5.571 \pm 6.578 vs. $3.812 \pm 4.307, \mathrm{P}=0.452)(\mathrm{B})$.

Before and during the process of hybridization, all equipment and materials were treated with $0.1 \%$ DEPC. All glassware was baked at $180^{\circ} \mathrm{C}$ for $4 \mathrm{~h}$ to destroy RNases. Formalin-fixed paraffin-embedded sections $(5 \mu \mathrm{m})$ were deparaffinized in three consecutive xylene baths for $5 \mathrm{~min}$ each, followed by 5 min each in serial dilutions of ethanol $(100,100,95,85$, $70 \%$ ) and three changes of $0.1 \%$ DEPC-treated water. Slides were then immersed in 4\% PFA for $20 \mathrm{~min}$ at room temperature, washed twice with DEPC-treated PBS and digested with $10 \mu \mathrm{g} /$ $\mathrm{ml}$ proteinase $\mathrm{K}$ (Roche) at $37^{\circ} \mathrm{C}$ for $15 \mathrm{~min}$. The digestion was stopped with $0.1 \%$ glycine in PBS, washed once with DEPC-treated water and the slides were fixed again with $4 \%$ PFA for $15 \mathrm{~min}$. Slides were then pre-hybridized in incubation chambers at $50^{\circ} \mathrm{C}$ for $3-4 \mathrm{~h}$, and then hybridized at the hybridization temperature $\left(\mathrm{Tm}\right.$ probe $\left.-21^{\circ} \mathrm{C}\right)$ in an oven overnight, using $20 \mathrm{nM}$ LNA-modified probes (Exigon). After hybridization, slides were washed twice in $2 \mathrm{X}$ SSC and 0.5X SSC for $30 \mathrm{~min}$ at the hybridization temperature, and the slides were incubated in blocking solution for $1 \mathrm{~h}$ at room temperature. An AP-conjugated anti-digoxingenin antibody (1093274,
Roche) was diluted with blocking solution to 1:1000 overnight at $4^{\circ} \mathrm{C}$, followed by three washes with alkaline phosphatase buffer at room temperature. Finally, the slides were incubated with developing solution, which consisted of NBT and BCIP in alkaline phosphatase buffer in the dark for 2-48 $\mathrm{h}$ at room temperature until a strong blue staining was observed, followed by three washes in TBST. Slides were mounted and visualized.

Statistical analysis. Wilcoxon matched pairs test was used to determine the difference in $h$ Biot 2 expression between the normal mucosa and primary tumor in the same patient. The Mann-Whitney U test was used to determine the difference in $h$ Biot 2 expression between the primary tumor and liver metastasis, as well as the difference between the relative value of $h$ Biot 2 expression and clinicopathological variables. Cox's proportional hazards model was used to estimate the relationship between $h$ Biot 2 expression and patient survival in univariate and multivariate analyses. The Kaplan-Meier method was used to calculate survival curves. Two-sided P-values of $<5 \%$ were considered to indicate statistical significance.

\section{Results}

The mean value of hBiot 2 expression in the normal mucosa, primary tumor and liver metastasis. The mean value of $h$ Biot2 expression in the primary tumors $(243.571 \pm 564.569)$ was higher than both the normal mucosa $(107.252 \pm 413.635$, $\mathrm{P}<0.0001)$ and liver metastasis $(42.002 \pm 40.809, \mathrm{P}=0.0002)$ samples. Thus the mean value of $h$ Biot 2 expression in the primary tumors increased markedly compared to the normal mucosa, and then went down to the lowest level in the liver metastasis samples (Fig. 1A).

The relative value of hBiot 2 expression in primary tumor in relation to clinicopathological variables and biological factors. We used the relative value of $h$ Biot 2 expression, i.e., the $h$ Biot 2 expression value in the primary tumor divided by the $h$ Biot 2 expression value in the corresponding normal mucosa, to analyze the relationship between $h \mathrm{Biot} 2$ and clinicopathological variables. As shown in Fig. $1 \mathrm{~B}, h$ Biot2 expression was significantly increased from stage II to III (3.057 \pm 4.179 vs. $5.571 \pm 6.578, P=0.018$ ). Since the expression in stages I and II was very similar $(3.413 \pm 4.580$ vs. $3.057 \pm 4.179$, $\mathrm{P}=0.368$ ), we combined stage I with II for a comparison with stage III, and a significant difference $(3.159 \pm 4.256$ vs. $5.193 \pm 6.256, \mathrm{P}=0.047$ ) was noted. Stage IV seemed to have lower expression than stage III, but the difference was not significant $(5.571 \pm 6.578$ vs. $3.812 \pm 4.307, \mathrm{P}=0.452)$. There were no relationships between $h$ Biot 2 expression and gender, age, tumor site, growth patterns or differentiation $(\mathrm{P}>0.05)$ (data not shown).

To analyze the impact of the $h$ Biot 2 relative expression value on the prognosis of the patients with primary tumors, we divided the patients into a low $(n=39)$ and a high $(n=108)$ expression group by using the lowest $25 \%$ expression value as a cut-off point. Univariate analysis showed that those with a high relative value of $h$ Biot 2 expression had a worse outcome compared with those with a low relative value $(\mathrm{P}=0.003$, Fig. 2). Multivariate analysis revealed that the relative value of $h$ Biot2 expression was still a prognostic indicator in the 


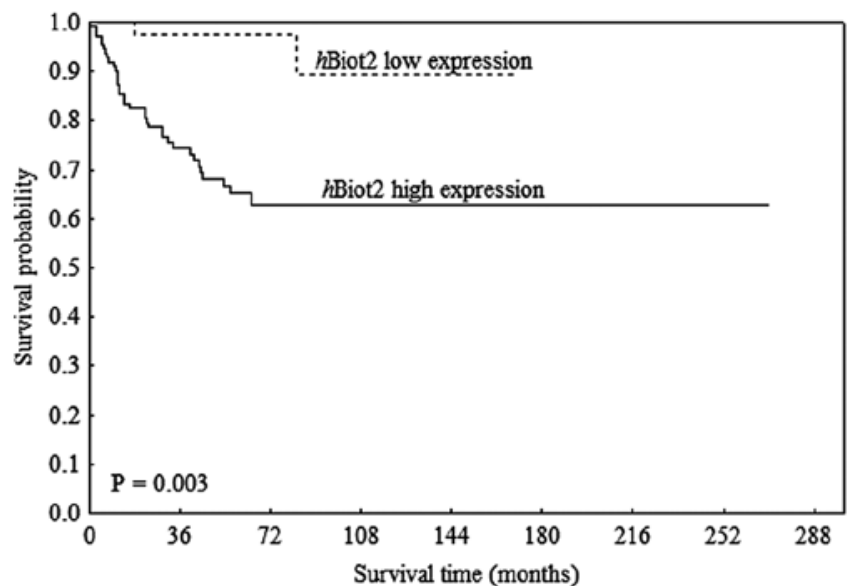

Figure 2. The relative value of $h$ Biot2 expression in the primary colorectal cancer patients was cut-off at the lowest $25 \%$ expression value for two groups; a low expression group $(\mathrm{n}=39)$ and a high expression group $(\mathrm{n}=108)$. The high expression group showed a worse outcome compared with the group with low expression $(\mathrm{P}=0.003)$.

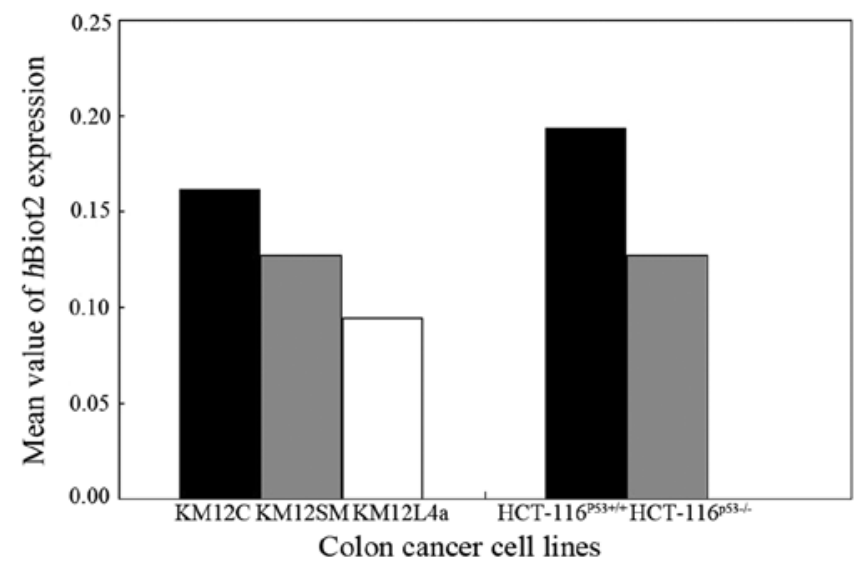

Figure 3 . There was an inverse relationship between the $h$ Biot 2 expression value and the metastasic potential of the three KM12 colon cancer cell lines. The metastasis potential increased from KM12C to KM12L4a, while the mean value of $h$ Biot2 expression decreased from KM12C to KM12L4a, In HCT-116, the metastasis potential in HCT-11 $6^{533-/ /}$ was stronger than HCT-11 $6^{\text {p53+/ }}$, while $h$ Biot2 expression value in HCT-11 $6^{\mathrm{p} 53-/-}$ was lower than in HCT-116 $6^{\mathrm{p} 53+/+}$.

Table I. Multivariate analysis of $h$ Biot 2 expression, gender, age, tumor site, stage and grade of differentiation in relation to survival in colorectal cancer patients.

\begin{tabular}{|c|c|c|c|c|}
\hline Variables & $\mathrm{n}$ & Cancer death rate ratio & $95 \%$ confidence interval & P-value \\
\hline$h$ Biot2 expression & & & & 0.0070 \\
\hline Low & 39 & 1.000 & - & \\
\hline High & 108 & 7.519 & $1.729-32.704$ & \\
\hline Gender & & & & 0.9220 \\
\hline Male & 92 & 1.000 & - & \\
\hline Female & 55 & 1.039 & $0.487-2.214$ & \\
\hline Age (years) & & & & 0.2590 \\
\hline$\leq 72$ & 61 & 1.000 & - & \\
\hline$>72$ & 86 & 1.510 & $0.738-3.088$ & \\
\hline Site & & & & 0.0390 \\
\hline Colon & 91 & 1.000 & - & \\
\hline Rectum & 56 & 0.432 & $0.195-0.959$ & \\
\hline Stage & & & & $0.0001^{\mathrm{a}}$ \\
\hline I & 29 & 1.000 & - & \\
\hline II & 74 & 1.289 & $0.278-5.923$ & \\
\hline III & 29 & 3.172 & $0.704-14.286$ & \\
\hline IV & 15 & 11.234 & $2.279-55.392$ & \\
\hline Differentiation & & & & 0.1810 \\
\hline Well & 105 & 1.000 & - & \\
\hline Poor & 42 & 1.696 & $0.783-3.674$ & \\
\hline
\end{tabular}

${ }^{\text {aTest for trend. }}$

patients, independently of gender, age, tumor site, stage and differentiation $(\mathrm{P}=0.007$, rate ratio $7.519,95 \%$ confidence interval 1.729-32.704, Table I). We compared the relative value of $h$ Biot 2 expression with the expression of p73 $(n=21)$ and MAC30 $(\mathrm{n}=29)$ in CRC. $h$ Biot 2 expression was positively related to the expression of $\mathrm{p} 73(\mathrm{P}=0.027)$ and MAC30 $(\mathrm{P}=0.002)$.

The mean value of hBiot2 expression in liver metastasis in relation to clinicopathological variables. Liver metastasis 
Table II. $h$ Biot 2 expression in relation to clinicopathological variables in the liver metastasis patients.

\begin{tabular}{lrrr}
\hline & \multicolumn{2}{c}{$h$ Biot2 } & \\
\cline { 2 - 3 } & $\mathrm{n}$ & Rank & P-value \\
\hline $\begin{array}{l}\text { Tumor size (mm) } \\
\quad \leq 42\end{array}$ & 22 & 499 & 0.003 \\
$\quad>42$ & 14 & 167 & \\
Local recurrence in the liver & & & 0.044 \\
$\quad$ No & 28 & 465 & \\
$\quad$ Yes & 8 & 201 & \\
Distant metastasis & & & 0.090 \\
besides the liver & & & \\
$\quad$ No & 30 & 595 & \\
$\quad$ Yes & 6 & 71 & \\
Liver surgery & & & 0.026 \\
$\quad$ Primary & 10 & 248 & \\
$\quad$ Repeated & 26 & 418 & \\
\hline
\end{tabular}

samples were separated according to the size of the largest metastasis. The mean value of $h$ Biot 2 expression in the group of the small-size tumors $(\leq 42 \mathrm{~mm})$ was higher than that in the group of the large-size tumors $(>42 \mathrm{~mm}, \mathrm{P}=0.003)$. The samples were then separated according to non-local or local recurrence in the liver. The mean value of $h \operatorname{Biot} 2$ expression in the non-local recurrence group was higher than that in the group having local recurrence in the liver $(\mathrm{P}=0.044)$. When the patients were separated based on whether they had primary or repeated liver surgery, the mean value of $h$ Biot2 expression was higher in the metastasis samples from the patients with primary surgery than those with repeated liver surgery $(\mathrm{P}=0.026)$. Some patients had metastases to other organs other than the liver (distant metastasis). The mean value of $h$ Biot 2 expression was lower in those having distant metastases when compared with patients without non-distant metastases, even though the difference did not reach statistical significance $(\mathrm{P}=0.090$, Table II). There were no significant differences between the mean value of $h$ Biot 2 expression in the liver metastasis samples and clinicopathological variables, such as, gender $(\mathrm{P}=0.873)$, age $(\mathrm{P}=0.327)$, number of metastasis $(\leq$ or $>2)$ in the liver $(\mathrm{P}=0.835)$, metastasis after/within 6 months after primary tumor diagnosis $(\mathrm{P}=0.889)$ and extra liver growth $(\mathrm{P}=0.167)$ (data not shown).

The mean value of hBiot 2 expression in colon cancer cell lines. The mean value of $h \mathrm{Biot} 2$ expression in $\mathrm{KM} 12 \mathrm{C}$, KM12SM, KM12L4a, HCT-116 $6^{\mathrm{p} 53+/+}$ and HCT-116 $6^{\mathrm{p} 33-/-}$ was $0.161,0.127,0.095,0.193$ and 0.127 , respectively. In the three KM cell lines, the mean value of $h$ Biot 2 expression was the highest in KM12C and the lowest in KM12L4a. In the two HCT-116 cell lines, the expression was higher in HCT-116 $6^{\mathrm{p} 53+/+}$ than in HCT-116 p53-- $^{\text {(Fig. 3). }}$.

RNA expression of hBiot 2 determined by ISH in CRC and normal mucosa. Fig. 4 shows RNA expression of $h$ Biot 2 determined by ISH in CRC and normal mucosa. $h$ Biot2 expression was strong in the nucleus of primary tumor cells (A) and normal epithelial cells (D), while stromal cells, such as fibroblasts, did not express $h$ Biot 2 . There was no staining in the negative control both in the tumors (B) and normal mucosa (E), while $\beta$-actin, as the positive control, was strongly expressed in the cytoplasm and nucleus of the tumors (C) and normal epithelial cells (F).

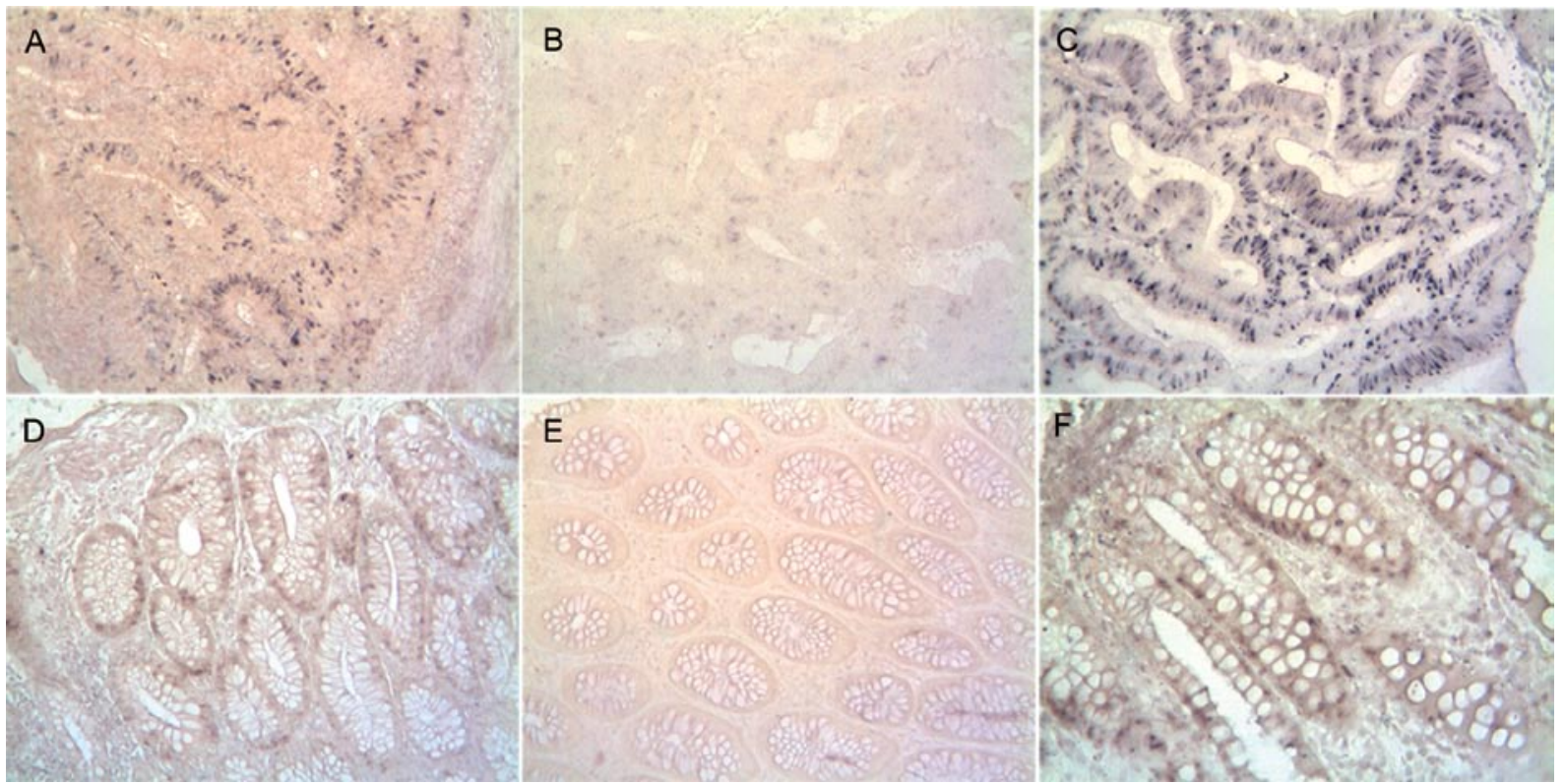

Figure 4. RNA expression of $h$ Biot 2 determined by in situ hybridization on formalin-fixed paraffin-embedded sections in colorectal cancer and normal mucosa (x200). $h$ Biot2 was expressed strongly in the nucleus of tumor (A) and normal epithelial cells (D). Negative control probe showed no staining both in the tumor cells (B) and normal mucosa (E). $\beta$-actin, as a positive control, was expressed strongly in the cytoplasm and nucleus of the tumor (C) and normal epithelial cells $(F)$. 


\section{Discussion}

The most important characteristic of cancer cells is the increased ability to proliferate and the decreased susceptibility to apoptosis (20). Moreover, studying the expression profile of a novel gene in tissues may help to determine the function of the gene and better understand the mechanism of carcinogenesis and cancer progression (21-23). In previous studies, we identified that the $r$ Biot 2 gene stimulated proliferation of normal sperm cells in rat and mouse $(7,8)$. In a further study of human endometrial cancer, we found that $h$ Biot 2 expression was higher in endometrial cancer than in the corresponding normal endometrium, and the expression was higher in poorly differentiated tumors compared with well-differentiated ones (9). The results indicated that $h$ Biot 2 may have a potential function in the development of endometrial cancer. Therefore, we wanted to investigate the $h$ Biot 2 expression in CRC and its relationships with clinicopathological variables, as this has not been previously investigated.

In the present study, $h$ Biot 2 expression was higher in the primary tumors compared with either the corresponding normal mucosa or liver metastasis samples, while the lowest expression was in liver metastasis. We further found that $h$ Biot 2 expression was increased from stages I/II to stage III, while there was no significant difference in $h$ Biot2 expression between stages III and IV. It seemed that there was a trend of increasing $h$ Biot 2 expression during the early and interim development of CRC, and then decreased in the late period of CRC development. In support of this finding, in the liver metastasis, we further noted that $h$ Biot 2 expression was lower in larger tumors versus smaller ones, local recurrence (in the liver) versus non-local recurrence, and primary liver surgery alone versus repeated liver surgery. In addition, we evaluated the $h$ Biot2 expression value in colon cancer cell lines, KM12C, KM12SM, KM12L4a, HCT-116 $6^{\text {p53+/ }}$ and HCT-116 ${ }^{\text {p53-- }}$. The $h$ Biot2 expression in KM12SM or KM12L4a was lower than in KM12C, while both KM12SM and KM12L4a have higher potential of metastasis than KM12C. The $h$ Biot 2 expression was lower in HCT-116 $6^{\mathrm{p} 33-/}$ compared to HCT-116 $6^{\mathrm{p} 33+/ \mathrm{t}}$. Tumors with mutant p53 (such as p53 $3^{-1}$ ), which is a late event in CRC development, have more malignant features than wild-type p53 $\left(\mathrm{p} 53^{+/+}\right)(24,25)$. Taken together, the expression value of $h$ Biot2 increased from the normal mucosa to primary tumor, and then decreased with progression of either primary tumor or liver metastasis.

We further investigated whether $h$ Biot 2 expression in CRCs was related to patient survival. Higher expression of $h$ Biot2 was related to a worse prognosis independently of gender, age, tumor site, stage and differentiation.

To establish the localization of the $h$ Biot 2 gene expressed in the primary tumor and normal mucosa, we examined the RNA expression of $h$ Biot 2 on formalin-fixed paraffin-embedded sections from primary CRCs and normal mucosa by ISH. Our results showed that $h$ Biot 2 was expressed in both tumor cells and normal epithelial cells, but not in the stromal cells.

In order to better understand the role of $h$ Biot 2 in the development of CRC and the pathway involved, we also investigated the relationships of $h \mathrm{Biot} 2$ with $\mathrm{p} 73$ and MAC 30 $(10,11)$ expression (data from the same material used in the present study). We found that $h \operatorname{Biot} 2$ expression was positively related to both $\mathrm{p} 73$ and MAC30. Overexpression of $\mathrm{p} 73$ protein has been correlated with a poor prognosis in colorectal, hepatocellular and breast cancers $(26,27)$. In the same samples, we previously found that p73 was overexpressed in colorectal cancer compared with normal mucosa. The patients with p73-overexpressing tumors tended to have a higher local recurrence after radiotherapy compared to non-radiotherapy and a poor prognosis $(10,27)$. The expression of MAC30 was found to be stronger in breast, stomach and CRCs than the corresponding normal mucosa $(11,28,29)$, indicating that MAC30 may act as an oncogene in cancers and may play a role in tumor development and aggressiveness. Taken together, the positive relationship of $h$ Biot 2 expression with p73 and MAC30 expression indicates that $h$ Biot 2 may interact with factors involved in cell proliferation, apoptosis and invasiveness of CRC.

In conclusion, the increased expression of $h$ Biot 2 may be an early and interim event in the development of CRC. High expression of $h$ Biot 2 independently indicates a worse prognosis in primary CRC patients.

\section{Acknowledgements}

The authors are grateful to Dr David Hinselwood for the linguistic revision. This study was supported by grants from the Swedish Cancer Foundation, Swedish Research Council and the Health Research Council in Southeast Sweden.

\section{References}

1. Stewart BW and Kleihues P: World Cancer Report. World Health Organization, WHO Press, 2003.

2. Cancerfonden: Cancerfondsrapporten, 2008 (In Swedish),

3. Duffy MJ, van Dalen A, Haglund C, et al: Clinical utility of biochemical markers in colorectal cancer: European Group on Tumour Marker (EGTM) guidelines. Eur J Cancer 39: 718-727, 2003.

4. Anwar S, Frayling IM, Scott NA, et al: Systematic review of genetic influences on the prognosis of colorectal cancer. Br J Surg 91: 1275-1291, 2004

5. Miyoshi N, Ishii H, Sekimoto M, Doki Y and Mori M: RGS16 is a marker for prognosis in colorectal cancer. Ann Surg Oncol 16: 3507-3514, 2009.

6. Chen YT, Scanlan MJ, Sahin U, et al: A testicular antigen aberrantly expressed in human cancers detected by autologous antibody screening. Proc Natl Acad Sci USA 94: 1914-1918, 1997.

7. Yang H, Wang C, Wang R, et al: Characterization of a novel rat gene RTAP2a, screened by cross-reactive SEREX, restrictedly expressed in testis. J Biosci Bioeng 107: 589-595, 2009.

8. Wang CT, Zhang P, Wang YS, et al: RNA interference against Biot2, a novel mouse testis-specific gene, inhibits the growth of tumor cells. Cell Mol Biol Lett 14: 363-376, 2009.

9. Yangmei S, Xiang H, Hongxin D, et al: Expression of human Biot 2 and its potential function on carcinogenesis in endometrial cancer. Acta Obstet Gynecol Scand 86: 1503-1509, 2007.

10. Pfeifer D, Gao J, Adell G and Sun XF: Expression of the p73 protein in rectal cancers with or without preoperative radiotherapy. Int J Radiat Oncol Biol Phys 65: 1143-1148, 2006.

11. Zhang ZY, Zhao ZR, Adell G, et al: Expression of MAC30 in rectal cancers with or without preoperative radiotherapy. Oncology 71: 259-265, 2006.

12. Morikawa K, Walker SM, Nakajima M, Pathak S, Jessup JM and Fidler IJ: Influence of organ environment on the growth, selection, and metastasis of human colon carcinoma cells in nude mice. Cancer Res 48: 6863-6871, 1988.

13. Morikawa K, Walker SM, Jessup JM and Fidler IJ: In vivo selection of highly metastatic cells from surgical specimens of different primary human colon carcinomas implanted into nude mice. Cancer Res 48: 1943-1948, 1988. 
14. Murray-Zmijewski F, Lane DP and Bourdon JC: p53/p63/p73 isoforms: an orchestra of isoforms to harmonise cell differentiation and response to stress. Cell Death Differ 13: 962-972, 2006.

15. Nakamura Y, Nawata M and Wakitani S: Expression profiles and functional analyses of Wnt-related genes in human joint disorders. Am J Pathol 167: 97-105, 2005.

16. Mori M, Staniunas RJ, Barnard GF, Jessup JM, Steele GD Jr and Chen LB: The significance of carbonic anhydrase expression in human colorectal cancer. Gastroenterology 105: 820-826, 1993.

17. Petersen M, Nielsen CB, Nielsen KE, et al: The conformation of locked nucleic acids (LNA). J Mol Recognit 13: 44-53, 2003.

18. Bondensgaard K, Petersen M, Singh SK, et al: Structural studies of LNA: RNA duplexes by NMR: conformations and implications for RNase H activity. Chemistry 6: 2687-2695, 2000.

19. Petersen M, Nielsen JT, Bondensgaard K, Wengel J and Jacobsen JP: Structural characterization of LNA and alphaL-LNA hybridized to RNA. Nucleosides Nucleotides Nucleic Acids 22: 1691-1693, 2003.

20. Green DR and Evan GI: A matter of life and death. Cancer Cell 1: 19-30, 2002

21. Chen YC, Davidson B, Cheng CC, et al: Identification and characterization of membralin, a novel tumor-associated gene, in ovarian carcinoma. Biochim Biophys Acta 1730: 96-102, 2005.
22. Korkmaz CG, Korkmaz KS, Kurys P, et al: Molecular cloning and characterization of STAMP2, an androgen-regulated six transmembrane protein that is overexpressed in prostate cancer. Oncogene 24: 4934-4945, 2005.

23. Simon I, Zhuo S, Corral L, et al: B7-h4 is a novel membranebound protein and a candidate serum and tissue biomarker for ovarian cancer. Cancer Res 66: 1570-1575, 2006.

24. Katkoori VR, Jia X, Shanmugam C, et al: Prognostic significance of p53 codon 72 polymorphism differs with race in colorectal adenocarcinoma. Clin Cancer Res 15: 2406-2416, 2009.

25. Pancione M, Forte N, Fucci A, et al: Prognostic role of betacatenin and p53 expression in the metastatic progression of sporadic colorectal cancer. Hum Pathol 41: 867-876, 2010.

26. Bénard J, Douc-Rasy S and Ahomadegbe JC. TP53 family members and human cancers. Hum Mutat 21: 182-191, 2003.

27. Sun XF: p73 overexpression is a prognostic factor in patients with colorectal adenocarcinoma. Clin Cancer Res 8: 165-170, 2002.

28. Kayed H, Kleeff J, Ding J, et al: Expression analysis of MAC30 in human pancreatic cancer and tumors of the gastrointestinal tract. Histol Histopathol 19: 1021-1031, 2004.

29. Moparthi SB, Arbman G, Wallin A, et al: Expression of MAC30 protein is related to survival and biological variables in primary and metastatic colorectal cancers. Int J Oncol 30: 91-95, 2007. 